\title{
Collins fragmentation function for pions and kaons in a spectator model
}

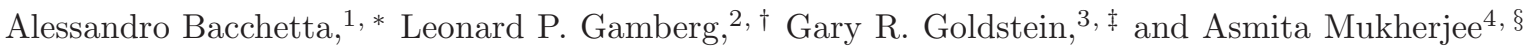 \\ ${ }^{1}$ Theory Group, Deutsches Elektronen-Synchroton DESY, \\ D-22603 Hamburg, Germany \\ ${ }^{2}$ Department of Physics, Penn State University, Berks, \\ Reading, PA 19610, USA \\ ${ }^{3}$ Department of Physics and Astronomy, Tufts University, \\ Medford, MA 02155, USA \\ ${ }^{4}$ Physics Department, Indian Institute of Technology Bombay, \\ Powai, Mumbai 400076, India
}

\begin{abstract}
We calculate the Collins fragmentation function in the framework of a spectator model with pseudoscalar pion-quark coupling and a Gaussian form factor at the vertex. We determine the model parameters by fitting the unpolarized fragmentation function for pions and kaons. We show that the Collins function for the pions in this model is in reasonable agreement with recent parametrizations obtained by fits of the available data. In addition, we compute for the first time the Collins function for the kaons.

PACS numbers: 13.60.Le,13.87.Fh,12.39.Fe
\end{abstract}

\section{INTRODUCTION}

The Collins fragmentation function [1] measures how the orientation of the quark spin influences the direction of emission of hadrons in the fragmentation process and can thus be used as a quark spin analyzer. It contributes to several single spin asymmetries (SSA) in hard processes, such as semi inclusive deep inelastic scattering (SIDIS), $p p$ collisions and $e^{+} e^{-}$annihilation into hadrons. We shall henceforth use the term "Collins asymmetries" to denote any asymmetry where the Collins function plays a role.

The first experimental evidence of a nonzero Collins function for pions came from the measurement of a Collins asymmetry in SIDIS on a proton target by the HERMES collaboration [2]. The same asymmetry, but on a deuteron target, was found to be consistent with zero by the COMPASS collaboration [3]. At the moment, the most convincing evidence of a nonzero pion Collins function comes from the measurements of a Collins asymmetry in $e^{+} e^{-}$annihilation [4]. First extractions of the pion Collins function were performed in Ref. 5, 6]. A recent fit to SIDIS and $e^{+} e^{-}$ annihilation allowed the simultaneous extraction of the Collins fragmentation function and of the transversity parton distribution function [7], clearly showing the importance of the Collins function as a tool to investigate the structure of hadrons. The kaon Collins function is at the moment unknown.

A few model calculations of the Collins function for pions have been presented in the literature [8, 9, 10, 11, 12] and used to make predictions and/or compare to available data [10, 13, 14, 15]. However, the above calculations have been found to be inadequate to describe the data.

The aim of the present work is to show that a Collins function in reasonable agreement with the available parametrizations can be obtained in a model with pseudoscalar pion-quark coupling and Gaussian form factors at the pion-quark vertex. We also present, for the first time, the Collins function for the fragmentation of quarks into kaons. This calculation is relevant for the interpretation of recent kaon measurements done at HERMES [16] as well as COMPASS [17] and for future measurements at BELLE and JLab.

\footnotetext{
*Electronic address: alessandro.bacchetta@desy.de

${ }^{\dagger}$ Electronic address: lpg10@psu.edu

‡Electronic address: gary.goldstein@tufts.edu

§Electronic address: asmita@phy.iitb.ac.in
} 


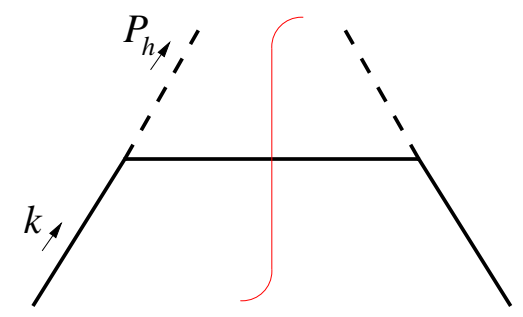

FIG. 1: Tree-level diagram for quark to meson fragmentation process.

\section{MODEL CALCULATION OF THE UNPOLARIZED FRAGMENTATION FUNCTION}

In the fragmentation process, the probability to produce hadron $h$ from a transversely polarized quark $q$, in, e.g., the $q \bar{q}$ rest frame if the fragmentation takes place in $e^{+} e^{-}$annihilation, is given by (see, e.g., [18])

$$
D_{h / q^{\uparrow}}\left(z, K_{T}^{2}\right)=D_{1}^{q}\left(z, K_{T}^{2}\right)+H_{1}^{\perp q}\left(z, K_{T}^{2}\right) \frac{\left(\hat{\boldsymbol{k}} \times \boldsymbol{K}_{T}\right) \cdot \boldsymbol{s}_{q}}{z M_{h}},
$$

where $M_{h}$ the hadron mass, $k$ is the momentum of the quark, $s_{q}$ its spin vector, $z$ is the light-cone momentum fraction of the hadron with respect to the fragmenting quark, and $K_{T}$ the component of the hadron's momentum transverse to k. $D_{1}^{q}$ is the unintegrated unpolarized fragmentation function, while $H_{1}^{\perp q}$ is the Collins function. Therefore, $H_{1}^{\perp q}>0$ corresponds to a preference of the hadron to move to the left if the quark is moving away from the observer and the quark spin is pointing upwards.

In accordance with factorization, fragmentation functions can be calculated from the correlation function [19]

$$
\begin{aligned}
\Delta\left(z, k_{T}\right) & =\frac{1}{2 z} \int d k^{+} \Delta\left(k, P_{h}\right) \\
& =\left.\frac{1}{2 z} \sum_{X} \int \frac{d \xi^{+} d^{2} \boldsymbol{\xi}_{T}}{(2 \pi)^{3}} e^{i k \cdot \xi}\left\langle 0\left|\mathcal{U}_{(+\infty, \xi)}^{n_{+}} \psi(\xi)\right| h, X\right\rangle\left\langle h, X\left|\bar{\psi}(0) \mathcal{U}_{(0,+\infty)}^{n_{+}}\right| 0\right\rangle\right|_{\xi^{-}=0},
\end{aligned}
$$

with $k^{-}=P_{h}^{-} / z$. A discussion on the structure of the Wilson lines, $\mathcal{U}$, can be found in Ref. [19]. Here, we limit ourselves to recalling that in Refs. [20, 21] it was shown that the fragmentation correlators are the same in both semiinclusive DIS and $e^{+} e^{-}$annihilation, as was also observed earlier in the context of a specific model calculation [20] similar to the one under consideration here. In the rest of the article we shall utilize the Feynman gauge, in which transverse gauge links at infinity give no contribution and can be neglected [22, 23, 24].

The tree-level diagram describing the fragmentation of a virtual (timelike) quark into a pion/kaon is shown in Fig. 1. In the model used here, the final state $|h, X\rangle$ is described by the detected pion/kaon and an on-shell spectator, with the quantum numbers of a quark and with mass $m_{s}$. We take a pseudoscalar pion-quark coupling of the form $g_{a \pi} \gamma_{5} \tau_{i}$, where $\tau_{i}$ are the generators of the $\mathrm{SU}(3)$ flavor group. Our model is similar to the ones used in, e.g., Refs. [25, 26, 27, 28]. The most important difference from previous calculations that included also the Collins function, i.e., those in Refs. [8, 19, 10, 11, 12], is that the mass of the spectator $m_{s}$ is not constrained to be equal to the mass of the fragmenting quark.

The fragmentation correlator at tree level, for the case $u \rightarrow \pi^{+}$, is

$$
\Delta_{(0)}(k, p)=-\frac{2 g_{q \pi}^{2}}{(2 \pi)^{4}} \frac{(\not k+m)}{k^{2}-m^{2}} \gamma_{5}\left(\not k-\not P_{h}+m_{s}\right) \gamma_{5} \frac{(\not k+m)}{k^{2}-m^{2}} 2 \pi \delta\left(\left(k-P_{h}\right)^{2}-m_{s}^{2}\right)
$$

and, using the $\delta$-function to perform the $k^{+}$integration,

$$
\Delta_{(0)}\left(z, k_{T}\right)=\frac{2 g_{q \pi}^{2}}{32 \pi^{3}} \frac{(\not k+m)\left(\not k-\not p_{h}-m_{s}\right)(\not k+m)}{(1-z) P_{h}^{-}\left(k^{2}-m^{2}\right)^{2}},
$$

where $k^{2}$ is related to $k_{T}^{2}$ through the relation

$$
k^{2}=z k_{T}^{2} /(1-z)+m_{s}^{2} /(1-z)+M_{h}^{2} / z
$$


which follows from the on-mass-shell condition of the spectator quark of mass $m_{s}$. We take $m$ to be the same for $u$ and $d$ quarks, but different for $s$ quarks. Isospin and charge-conjugation relations imply

$$
\begin{aligned}
D_{1}^{u \rightarrow \pi^{+}}=D_{1}^{\bar{d} \rightarrow \pi^{+}} & =D_{1}^{d \rightarrow \pi^{-}}=D_{1}^{\bar{u} \rightarrow \pi^{-}}, \\
D_{1}^{u \rightarrow K^{+}} & =D_{1}^{\bar{u} \rightarrow K^{-}} \\
D_{1}^{\bar{s} \rightarrow K^{+}} & =D_{1}^{s \rightarrow K^{-}} .
\end{aligned}
$$

For later purposes it is useful to spell out the relations coming from isospin and charge-conjugation relations for the unfavored functions

$$
\begin{aligned}
& D_{1}^{\bar{u} \rightarrow \pi^{+}}=D_{1}^{d \rightarrow \pi^{+}}=D_{1}^{\bar{d} \rightarrow \pi^{-}}=D_{1}^{u \rightarrow \pi^{-}}, \\
& D_{1}^{s \rightarrow \pi^{+}}=D_{1}^{\bar{s} \rightarrow \pi^{+}}=D_{1}^{s \rightarrow \pi^{-}}=D_{1}^{\bar{s} \rightarrow \pi^{-}}, \\
& D_{1}^{\bar{u} \rightarrow K^{+}}=D_{1}^{\bar{d} \rightarrow K^{+}}=D_{1}^{d \rightarrow K^{+}}=D_{1}^{\bar{d} \rightarrow K^{-}}=D_{1}^{d \rightarrow K^{-}}=D_{1}^{u \rightarrow K^{-}} \text {, } \\
& D_{1}^{s \rightarrow K^{+}}=D_{1}^{\bar{s} \rightarrow K^{-}} \text {. }
\end{aligned}
$$

We assume the above relations hold for all fragmentation functions, in particular for the Collins function.

The unpolarized fragmentation function is projected from Eq. (4)

$$
D_{1}\left(z, k_{T}^{2}\right)=\operatorname{Tr}\left[\Delta_{0}\left(z, k_{T}\right) \gamma^{+}\right] / 2
$$

leading to the result

$$
D_{1}\left(z, k_{T}^{2}\right)=\frac{g_{q \pi}^{2}}{8 \pi^{3}} \frac{\left[z^{2} \boldsymbol{k}_{T}^{2}+\left(z m+m_{s}-m\right)^{2}\right]}{z^{3}\left(\boldsymbol{k}_{T}^{2}+L^{2}\right)^{2}},
$$

with

$$
L^{2}=\frac{(1-z)}{z^{2}} M_{h}^{2}+m^{2}+\frac{m_{s}^{2}-m^{2}}{z}
$$

In the limit $m_{s}=m$ and setting the form factor to 1, our result for $D_{1}$ reduces to Eq. (3) of Ref. [12] (multiplied by two because in that paper the results refer to $\left.u \rightarrow \pi^{0}\right)$. The two nonzero kaon fragmentation functions $D_{1}^{u \rightarrow K^{+}}$and $D_{1}^{\bar{s} \rightarrow K^{+}}$are given by the same functional form, but with different masses $m, m_{s}, M_{h}$.

The integrated unpolarized fragmentation function is defined as

$$
D_{1}(z)=\pi z^{2} \int_{0}^{\infty} d k_{T}^{2} D_{1}\left(z, k_{T}^{2}\right) .
$$

Here the integration is over the transverse momentum of the produced hadron $K_{T}=-z k_{T}$ with respect to the quark direction, which is why an extra factor of $z^{2}$ appears in the above equation. The above integral is divergent. In Ref. [12], a cutoff on $k_{T}$ has been used. On the other hand, in Ref. [10], a Gaussian form factor depending on $k_{T}^{2}$ has been introduced at the pion-quark vertex, which effectively cuts off the higher $k_{T}$ region in the integration. Similarly, in this work we use a Gaussian form factor of the form

$$
g_{q \pi} \mapsto \frac{g_{q \pi}}{z} e^{-\frac{k^{2}}{\Lambda^{2}}} \quad \text { with } \quad \Lambda^{2}=\lambda^{2} z^{\alpha}(1-z)^{\beta} .
$$

at the pion-quark vertex. Due to Eq. (5), the above form factor effectively cuts off the higher $k_{T}$ region of the integration. The form of the vertex is chosen merely on the basis of phenomenological motivations, in order to reasonably reproduce the unpolarized fragmentation function. With the chosen form factor, the integration in Eq. (16) can be carried out analytically and yields:

$$
\begin{aligned}
D_{1}(z)=\frac{g_{q \pi}^{2}}{8 \pi^{2}} \frac{e^{-\frac{2 m^{2}}{\Lambda^{2}}}}{z^{3} L^{2}}[ & (1-z)\left(\left(m_{s}-m\right)^{2}-M_{h}^{2}\right) \exp \left(-\frac{2 z L^{2}}{(1-z) \Lambda^{2}}\right) \\
& \left.+\left(z^{2} \Lambda^{2}-2 z\left(\left(m_{s}-m\right)^{2}-M_{h}^{2}\right)\right) \frac{L^{2}}{\Lambda^{2}} \Gamma\left(0, \frac{2 z L^{2}}{(1-z) \Lambda^{2}}\right)\right],
\end{aligned}
$$




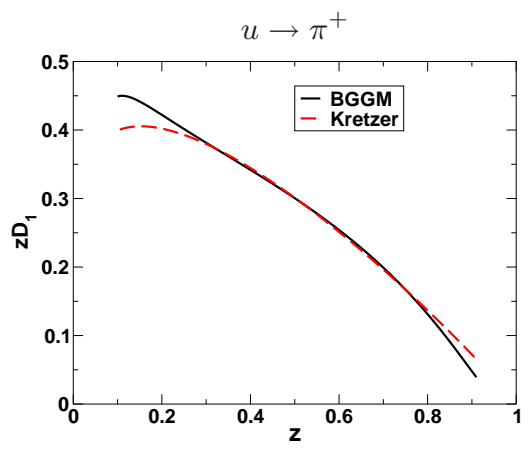

(a)

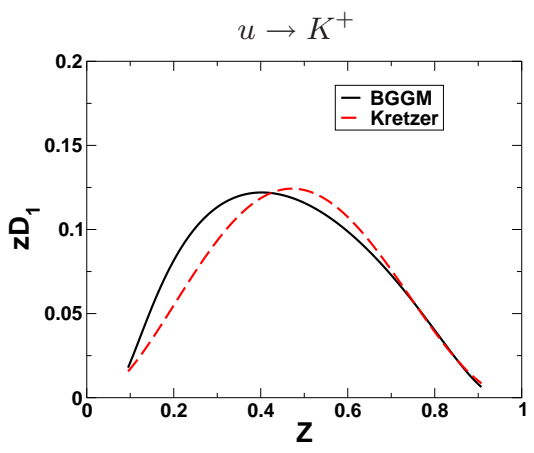

(b)

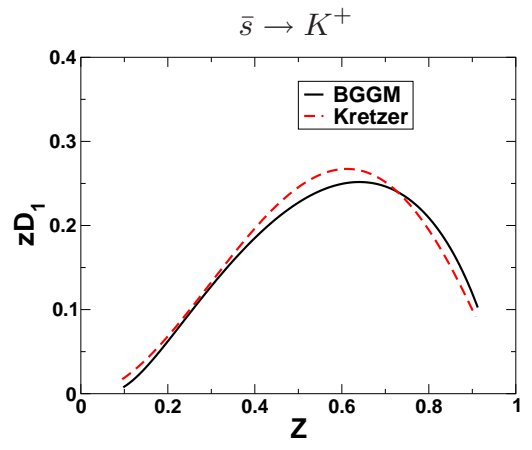

(c)

FIG. 2: Unpolarized fragmentation function $z D_{1}(z)$ vs. $z$ for the fragmentation (a) $u \rightarrow \pi^{+}$, (b) $u \rightarrow K^{+},(\mathrm{c}) \bar{s} \rightarrow K^{+}$in the spectator model (solid line), with parameters fixed from a fit to the parametrization of [29] (dashed line).

where the incomplete gamma function is,

$$
\Gamma(0, z) \equiv \int_{z}^{\infty} \frac{e^{-t}}{t} d t
$$

The parameters of the model are $\lambda, \alpha, \beta$, together with the mass of the spectator $m_{s}$ and the mass of the initial quark $m$. For the latter, we choose a constituent quark mass $m=0.3 \mathrm{GeV}$ for the $u$ and $d$ quarks, and $m=0.5$ $\mathrm{GeV}$ for the $s$ quark. To fix the values of the other parameters, we performed a fit to the parametrization of Ref. [29] (NLO set) at the lowest possible scale, i.e., $Q_{0}=0.4 \mathrm{GeV}^{2}$. The resulting values for the parameters are

$$
g_{q \pi}=4.78, \quad \lambda=3.33 \mathrm{GeV}, \quad \quad \alpha=0.5 \text { (fixed), } \quad \beta=0 \text { (fixed), }
$$

which are common to both pion and kaon fragmentation functions. The only parameters that change according to the type of fragmentation function are

$$
\begin{array}{rll}
u \rightarrow \pi^{+}: & m_{s}=0.792 \mathrm{GeV}, & m=0.3 \mathrm{GeV} \text { (fixed), } \\
u \rightarrow K^{+}: & m_{s}=1.12 \mathrm{GeV}, & m=0.3 \mathrm{GeV} \text { (fixed), } \\
\bar{s} \rightarrow K^{+}: & m_{s}=0.559 \mathrm{GeV}, & m=0.5 \mathrm{GeV} \text { (fixed) }
\end{array}
$$

Obviously, also the mass of the hadron changes: we take $m_{h}=0.135 \mathrm{GeV}$ for the pions and $m_{h}=0.494 \mathrm{GeV}$ for the kaons. We remark that it is not possible to estimate the errors in the parameters in a meaningful way because the fragmentation functions in Ref. 29] have no error bands. It could be in principle possible to use the recent parametrizations with error bands [30], but the lowest scale they reach is $1 \mathrm{GeV}^{2}$, which we consider to be too high to compare to our model.

Fig. 2 show the plots of the unpolarized fragmentation function $D_{1}(z)$ multiplied by $z$ for $u \rightarrow \pi^{+}, u \rightarrow K^{+}$, and $\bar{s} \rightarrow K^{+}$. The parametrization of [29] (NLO set, $Q_{0}=0.4 \mathrm{GeV}^{2}$ ) is also shown for comparison.

\section{MODEL CALCULATION OF THE COLLINS FRAGMENTATION FUNCTION}

We use the following definition of the Collins function $[12]^{1}$

$$
\frac{\epsilon_{T}^{i j} k_{T j}}{M_{h}} H_{1}^{\perp}\left(z, k_{T}^{2}\right)=\frac{1}{2} \operatorname{Tr}\left[\Delta\left(z, k_{T}\right) i \sigma^{i-} \gamma_{5}\right] .
$$

As is well known [12], using the tree-level calculation of the correlator function is not sufficient to produce a nonvanishing Collins function, due to the lack of imaginary parts in the scattering amplitude. In order to obtain the necessary imaginary part, we take into account gluon loops. In fact, gluon exchange is essential to ensure color gauge

\footnotetext{
1 The factor $1 / 2$ is due to a slightly different definition of the correlator in Eq. (2) with respect to Ref. [12]
} 


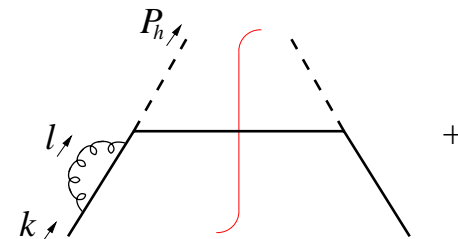

(a)

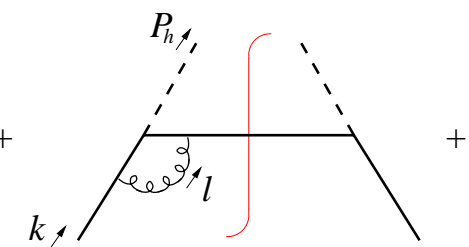

(b)

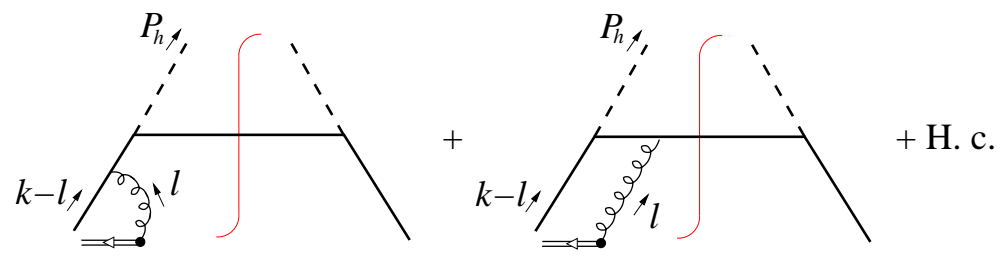

(c)

(d)

FIG. 3: Single gluon-loop corrections to the fragmentation of a quark into a pion contributing to the Collins function in the eikonal approximation. "H.c." stands for the hermitian conjugate diagrams which are not shown.

invariance of the fragmentation functions. Contributions come from the four diagrams in Fig. 3 Diagrams (a) and (b) represent the quark self-energy and vertex diagrams, respectively. Diagrams (c) and (d) can be called hard-vertex and box diagrams, respectively. For the calculation of the diagrams with the eikonal line, the Feynman rules to be used are

$$
\stackrel{\xi}{=}=i g t^{a} \delta_{\nu}^{-}, \quad \stackrel{l}{=}=\frac{i}{-l^{-} \pm i \epsilon},
$$

where $g$ is the QCD coupling. Note that the sign of the $i \epsilon$ in the eikonal propagator is different for semi-inclusive deep inelastic scattering $(+)$ and $e^{+} e^{-}$annihilation $(-)$, but this does not influence the computation of the Collins function [20, 21]. The resulting formula for the fragmentation correlator corresponding to Fig. 3 (d) is

$$
\begin{aligned}
\Delta_{1(d)}(k, p)= & \frac{4 \alpha_{s}}{(2 \pi)^{3}} \frac{i(\not k+m)}{k^{2}-m^{2}} g_{q \pi} \gamma_{5}\left(\not k-\not P_{h}+m_{s}\right) 2 \pi \delta\left(\left(k-P_{h}\right)^{2}-m_{s}^{2}\right) \\
& \int \frac{d^{4} l}{(2 \pi)^{4}} \frac{i \gamma_{\mu} t^{a} i\left(\not k-\not P_{h}-\not+m_{s}\right) g_{q \pi} \gamma_{5} i(\not k-\not+m) i\left(-i g^{\mu-}\right)\left(i t^{a}\right)}{\left(\left(k-P_{h}-l\right)^{2}-m_{s}^{2}+i \varepsilon\right)\left((k-l)^{2}-m^{2}+i \varepsilon\right)\left(-l^{-} \pm i \varepsilon\right)\left(l^{2}-m_{g}^{2}+i \varepsilon\right)} .
\end{aligned}
$$

Note that one of the form factors $g_{q \pi}$ is inside the loop integral. When using a form factor as in Eq. (17), it would seem reasonable to replace $k^{2}$ with $(k-l)^{2}$. However, since the form factor is introduced to the purpose of cutting off the high- $k_{T}$ region, we prefer to maintain the form factor depending only on $k^{2}$, so that it can be pulled out of the integral and simplify the calculation. This choice is similar to imposing a sharp cutoff on $k^{2}$ — as done in Ref. [9, 11, 12] — and not on $(k-l)^{2}$.

The Collins function is given by (we take always $m_{g}=0$ )

$$
H_{1}^{\perp}\left(z, k_{T}^{2}\right)=-\frac{2 \alpha_{s} g_{q \pi}^{2}}{(2 \pi)^{4}} C_{F} \frac{e^{-\frac{2 k^{2}}{\Lambda^{2}}}}{z^{2}} \frac{M_{h}}{(1-z)} \frac{1}{k^{2}-m^{2}}\left(\tilde{H}_{1(a)}^{\perp}\left(z, k_{T}^{2}\right)+\tilde{H}_{1(b)}^{\perp}\left(z, k_{T}^{2}\right)+\tilde{H}_{1(d)}^{\perp}\left(z, k_{T}^{2}\right)\right),
$$

where the subscripts in the r.h.s. refer to the contributions from diagrams 3 (a), (b) and (d) plus their Hermitean conjugate, respectively. Diagram (c) gives no contribution to the Collins function. The separate contributions read, for the fragmentation of $u \rightarrow \pi^{+}$,

$$
\begin{gathered}
\tilde{H}_{1(a)}\left(z, k_{T}^{2}\right)=\frac{m}{k^{2}-m^{2}}\left(3-\frac{m^{2}}{k^{2}}\right) I_{1 g}, \\
\tilde{H}_{1(b)}\left(z, k_{T}^{2}\right)=2 m_{s} I_{2 g}, \\
\tilde{H}_{1(d)}\left(z, k_{T}^{2}\right)=\frac{1}{2 z \boldsymbol{k}_{\boldsymbol{T}}^{2}}\left\{-I_{34 g}\left(2 z m+m_{s}-m\right)+I_{2 g}\left[2 z m\left(k^{2}-m^{2}+M_{h}^{2}(1-2 / z)\right)\right.\right. \\
\left.\left.+\left(m_{s}-m\right)\left((2 z-1) k^{2}-M_{h}^{2}+m_{s}^{2}-2 z m\left(m+m_{s}\right)\right)\right]\right\} .
\end{gathered}
$$




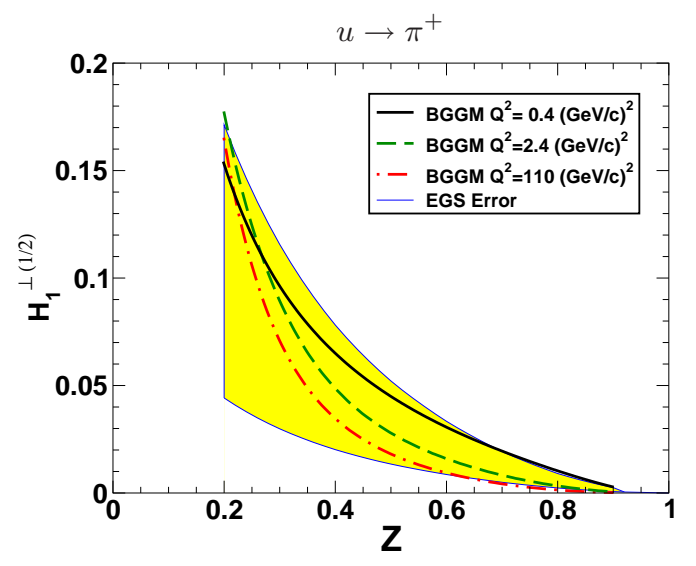

(a)

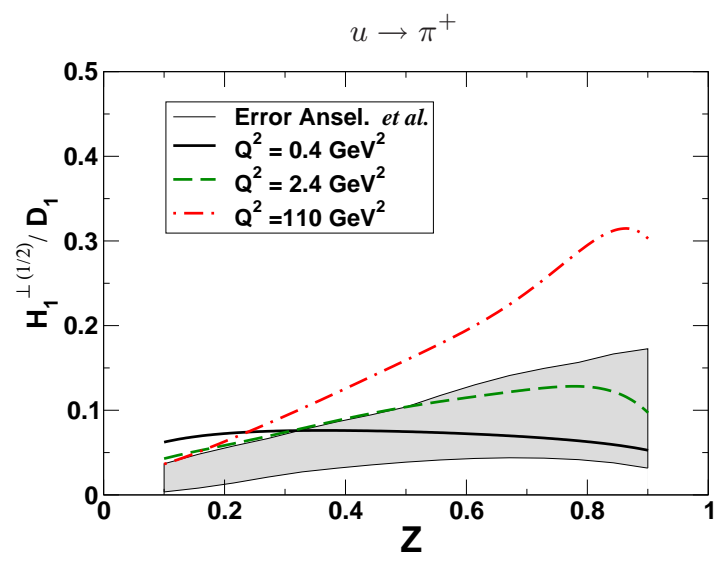

(b)

FIG. 4: Half moment of the Collins function for $u \rightarrow \pi^{+}$in our model. (a) $H_{1}^{\perp(1 / 2)}$ at the model scale (solid line) and at a different scale under the assumption in Eq. (37) (dot-dashed line), compared with the error band from the extraction of Ref. [6], (b) $H_{1}^{\perp(1 / 2)} / D_{1}$ at the model scale (solid line) and at two other scales (dashed and dot-dashed lines) under the assumption in Eq. (38). The error band from the extraction of Ref. [7] is shown for comparison.

The loop integrals $I_{1 g}, I_{2 g}$ and $I_{34 g}[12]^{2}$ are given by

$$
\begin{aligned}
I_{1 g} & =\frac{\pi \sqrt{\lambda\left(m, m_{g}\right)}}{2 k^{2}}, \\
I_{2 g} & =\frac{\pi}{2 \sqrt{\lambda\left(m_{s}, M_{h}\right)}} \ln \left[\frac{k^{2}+m_{s}^{2}-M_{h}^{2}-\sqrt{\lambda\left(m_{s}, M_{h}\right)}}{k^{2}+m_{s}^{2}-M_{h}^{2}+\sqrt{\lambda\left(m_{s}, M_{h}\right)}}\right], \\
I_{34 g} & =\pi \ln \left[\frac{\sqrt{k^{2}}(1-z)}{m_{s}}\right],
\end{aligned}
$$

where $\lambda\left(m_{1}, m_{2}\right)=\left(k^{2}-\left(m_{1}+m_{2}\right)^{2}\right)\left(k^{2}-\left(m_{1}-m_{2}\right)^{2}\right)$. In the limit $m_{s}=m$ and setting the form factor to 1 , our result for $H_{1}^{\perp}$ reduces to Eq. (15) of Ref. [12] (multiplied by two because in that paper the results refer to $u \rightarrow \pi^{0}$ ). It is important to note that the Collins function should obey the positivity bound [9, 31]

$$
\frac{\left|\boldsymbol{k}_{T}\right|}{M_{h}} H_{1}^{\perp}\left(z, k_{T}^{2}\right) \leq D_{1}\left(z, k_{T}^{2}\right) .
$$

Integration over $k_{T}^{2}$ gives the simplified expression

$$
\frac{H_{1}^{\perp(1 / 2)}(z)}{D_{1}(z)} \leq \frac{1}{2}
$$

where the half moment of the Collins function is defined as

$$
H_{1}^{\perp(1 / 2)}(z)=\pi z^{2} \int_{0}^{\infty} d k_{T}^{2} \frac{\left|\boldsymbol{k}_{T}\right|}{2 M_{h}} H_{1}^{\perp}\left(z, k_{T}^{2}\right) .
$$

In Fig. (4) (a), we have plotted the half moment of the Collins functions vs. $\mathrm{z}$ for the case $u \rightarrow \pi^{+}$. In the same panel, we plotted the 1- $\sigma$ error band of the Collins function extracted in Ref. [6] from BELLE data, collected at a scale $Q^{2}=(10.52)^{2} \mathrm{GeV}^{2}$. In order to achieve a reasonable agreement with the phenomenology, we choose a value of the strong coupling constant $\alpha_{s}=0.2$. Such a value is particularly small, especially when considering that our model has been tuned to fit the function $D_{1}$ at a scale $Q_{0}^{2}=0.4 \mathrm{GeV}^{2}$, where standard NLO calculations give $\alpha_{s} \approx 0.57$ [29, 32]. In any case, the problem of the choice of $\alpha_{s}$ is intimately related with the problem of the evolution of the Collins function (see below).

\footnotetext{
2 The expression $I_{34 g}$ used here corresponds to Eq. (24) in Ref. [12] multiplied by $k^{-}$.
} 


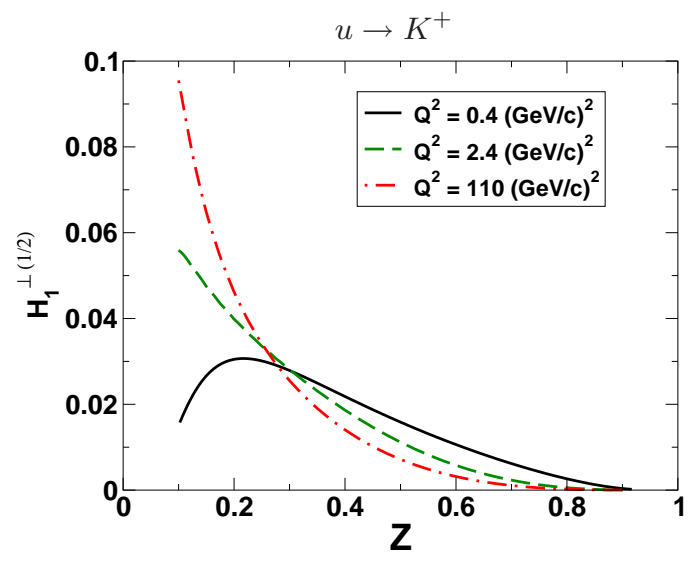

(a)

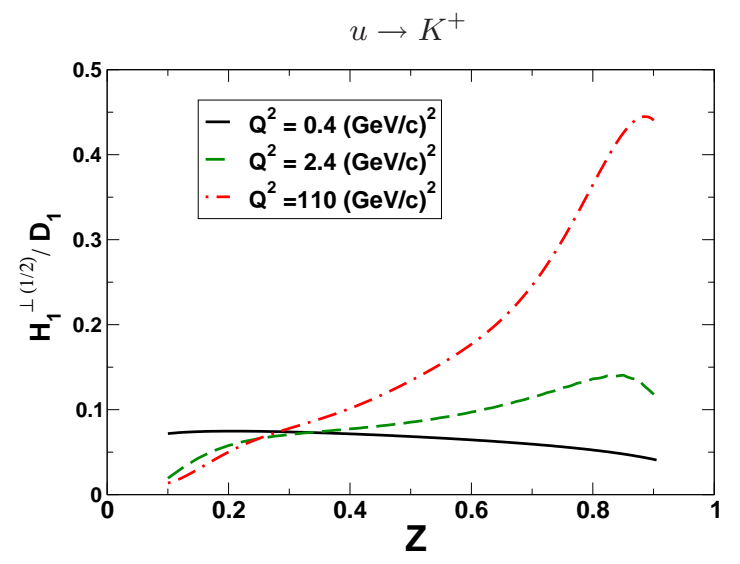

(b)

FIG. 5: Half moment of the Collins function for $u \rightarrow K^{+}$in our model. (a) $H_{1}^{\perp(1 / 2)}$ at the model scale of $0.4 \mathrm{GeV}^{2}$, (b) $H_{1}^{\perp(1 / 2)} / D_{1}$ at the model scale (solid line) and at two other scales (dashed and dot-dashed lines) under the assumption in Eq. (38).

In Fig. 4 (b), we have plotted the ratio $H_{1}^{\perp(1 / 2)} / D_{1}$ and compared it to the error bands of the extraction in Ref. [7]. Also in this case the agreement is good, with the above mentioned choice of $\alpha_{s}=0.2$.

At this point, some comments are in order concerning the evolution of the Collins function (or of its half-moment) with the energy scale. Such evolution is presently unknown, except for some work done in Ref. [33], which is however based on questionable assumptions. Some authors (e.g. Refs. [6, 7]) assume

$$
\left.\frac{H_{1}^{\perp(1 / 2)}}{D_{1}}\right|_{Q_{0}^{2}}=\left.\frac{H_{1}^{\perp(1 / 2)}}{D_{1}}\right|_{Q^{2}},
$$

i.e., that the evolution of $H_{1}^{\perp(1 / 2)}$ is equal to that of $D_{1}$. This seems unlikely, in view of the fact that the Collins function is chiral-odd and thus evolves as a non-singlet. An alternative choice could be to assume

$$
\left.H_{1}^{\perp(1 / 2)}\right|_{Q_{0}^{2}}=\left.H_{1}^{\perp(1 / 2)}\right|_{Q^{2}}
$$

i.e., that $H_{1}^{\perp(1 / 2)}$ does not evolve with the energy scale. This is an extreme hypothesis, which cannot be true because at some point the positivity bound (35) would be violated at large $z$. We demonstrate this in Fig. 4 (b) where we show how the ratio $H_{1}^{\perp(1 / 2)} / D_{1}$ behaves at at three different energy scales if only $D_{1}$ is evolved (we use the unpolarized fragmentation function of Ref. [29] for this purpose). Clearly, in this case the ratio grows more steeply with $z$ at higher energies, due to the decreasing of $D_{1}$ in the large- $z$ region. While the evolution of the T-odd parton distribution and fragmentation functions remain an outstanding issue, these results show that different assumptions on the Collins function scale dependence have a significant impact and should be considered with care.

For the fragmentation $u \rightarrow K^{+}$, the same analytic formulas are used but with the other set of parameter values.

\section{ASYMMETRIES IN $e^{+} e^{-}$ANNIHILATION}

The BELLE collaboration has reported measurements of various asymmetries in $e^{+}+e^{-} \rightarrow \pi^{ \pm}+\pi^{ \pm}+X$ that can isolate the Collins functions [4]. In particular, the number of pions in this case has an azimuthal dependence [34]

$$
\begin{aligned}
N_{h_{1} h_{2}}\left(z_{1}, z_{2}\right) \propto \sum_{q} e_{q}{ }^{2}( & D_{1\left(q \rightarrow h_{1}\right)}\left(z_{1}\right) D_{1\left(\bar{q} \rightarrow h_{2}\right)}\left(z_{2}\right) \\
& \left.+\frac{\sin ^{2} \theta}{1+\cos ^{2} \theta} \cos \left(\phi_{1}+\phi_{2}\right) H_{1\left(q \rightarrow h_{1}\right)}^{\perp(1 / 2)}\left(z_{1}\right) \bar{H}_{1\left(\bar{q} \rightarrow h_{2}\right)}^{\perp(1 / 2)}\left(z_{2}\right)\right),
\end{aligned}
$$

where $\phi_{1,2}$ are the azimuthal angles of the two pions relative to their jet axes (or thrust direction) and the 2 jet production plane. Normalizing this distribution and extracting the azimuthal asymmetry gives a measure of the 


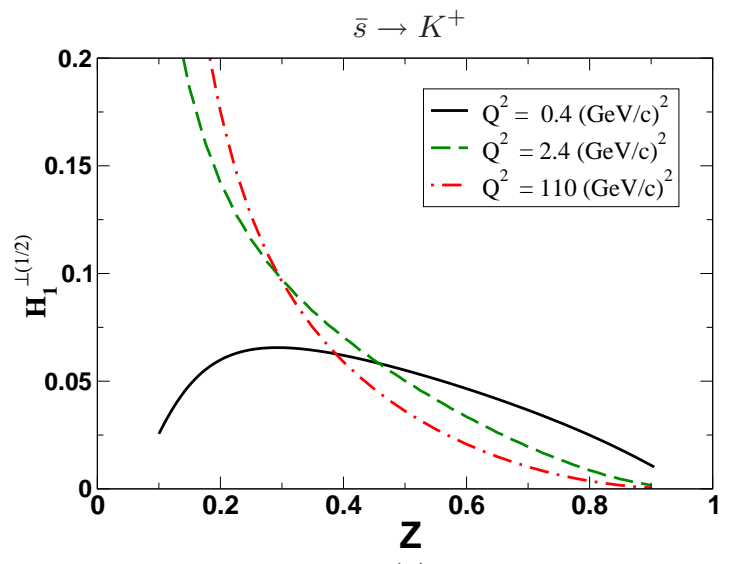

(a)

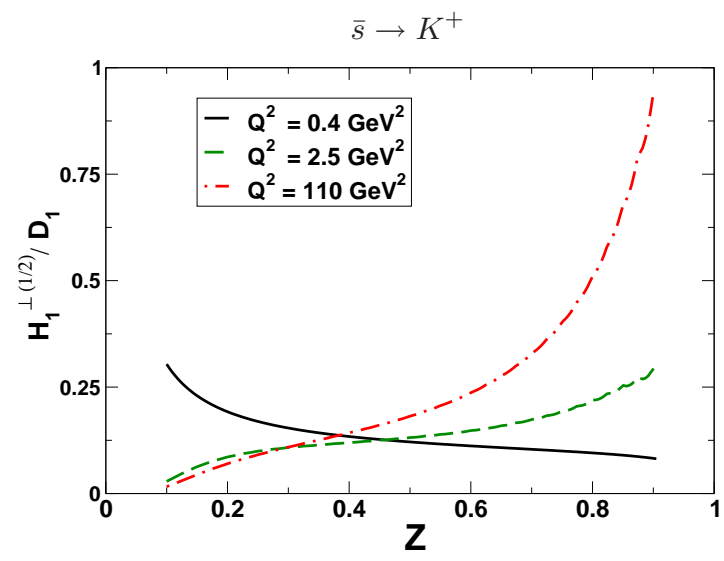

(b)

FIG. 6: Half moment of the Collins function for $\bar{s} \rightarrow K^{+}$in our model. (a) $H_{1}^{\perp(1 / 2)}$ at the model scale of $0.4 \mathrm{GeV}^{2}$, (b) $H_{1}^{\perp(1 / 2)} / D_{1}$ at the model scale (solid line) and at two other scales (dashed and dot-dashed lines) under the assumption in Eq. (38).

product of moments of Collins functions. BELLE noted that there are QCD radiative corrections that compete with the leading twist effects. To cancel out those corrections they take the ratio of the asymmetry for unlike sign events $\left(\pi^{+} \pi^{-}\right)$to the asymmetry for like sign events. This super ratio has the form [7]

$$
A_{12}\left(z_{1}, z_{2}\right)=\frac{\left\langle\sin ^{2} \theta\right\rangle}{\left\langle 1+\cos ^{2} \theta\right\rangle}\left(P_{U}-P_{L}\right)
$$

where

$$
\begin{aligned}
& P_{U}=\frac{\sum_{q} e_{q}^{2}\left(H_{1\left(q \rightarrow \pi^{+}\right)}^{\perp(1 / 2)}\left(z_{1}\right) H_{1\left(\bar{q} \rightarrow \pi^{-}\right)}^{\perp(1 / 2)}\left(z_{2}\right)+H_{1\left(q \rightarrow \pi^{-}\right)}^{\perp(1 / 2)}\left(z_{1}\right) H_{1\left(\bar{q} \rightarrow \pi^{+}\right)}^{\perp(1 / 2)}\left(z_{2}\right)\right)}{\sum_{q} e_{q}^{2}\left(D_{1\left(q \rightarrow \pi^{+}\right)}\left(z_{1}\right) D_{1\left(\bar{q} \rightarrow \pi^{-}\right)}\left(z_{2}\right)+D_{1\left(q \rightarrow \pi^{-}\right)}\left(z_{1}\right) D_{1\left(\bar{q} \rightarrow \pi^{+}\right)}\left(z_{2}\right)\right)},
\end{aligned}
$$

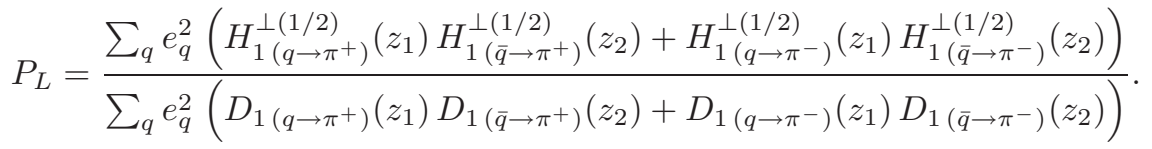

Note that Eq. (40) is a linear approximation for $P_{L}<<1$. For numerical studies, we take the unpolarized fragmentation functions from Ref. [29] (NLO set) at the scale of the BELLE measurements, i.e., $Q^{2}=(10.52)^{2} \mathrm{GeV}^{2}$. We take also $\left\langle\sin ^{2} \theta\right\rangle /\left\langle 1+\cos ^{2} \theta\right\rangle \approx 0.79$.

For the calculation of the asymmetry we have to make some assumptions on the unfavored Collins fragmentation functions. In order to have a guiding principle for our assumptions, we consider the Schäfer-Teryaev sum rule [35], which states that

$$
\sum_{h} \int_{0}^{1} d z H_{1(q \rightarrow h)}^{\perp(1)}(z)=0 \quad \text { with } \quad H_{1}^{\perp(1)}(z)=\pi z^{2} \int_{0}^{\infty} d k_{T}^{2} \frac{\boldsymbol{k}_{T}^{2}}{2 M_{h}^{2}} H_{1}^{\perp}\left(z, k_{T}^{2}\right) .
$$

We assume that the sum rule holds in a strong sense, i.e., for pions and kaons separately and at the integrand level, for each value of $z$ and $k_{T}$. For pions, it follows that

$$
H_{1\left(u \rightarrow \pi^{-}\right)}^{\perp(1 / 2)}=-H_{1\left(u \rightarrow \pi^{+}\right)}^{\perp(1 / 2)} .
$$

The other $\bar{u}, d, \bar{d}$, unfavored Collins functions are related to the above by isospin and charge symmetries, Eq. (9). Our strong interpretation of the Schäfer-Teryaev sum rule together with Eq. (10) (with $D_{1}$ replaced by $H_{1}^{\perp(1 / 2)}$ ) implies

$$
H_{1\left(s \rightarrow \pi^{-}\right)}^{\perp(1 / 2)}=-H_{1\left(s \rightarrow \pi^{+}\right)}^{\perp(1 / 2)}=0 .
$$




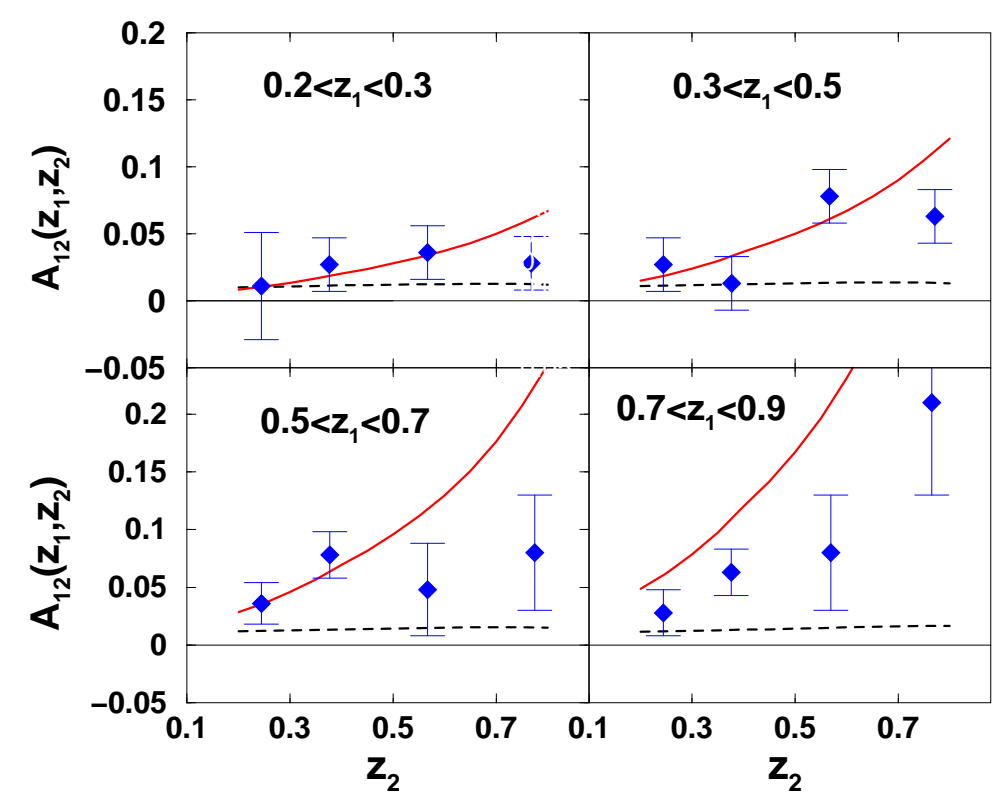

FIG. 7: Azimuthal asymmetry $A_{12}\left(z_{1}, z_{2}\right)$ for the production of two pions as a function of $z_{2}$ and integrated in bins of $z_{1}$ at $Q^{2}=110.7 \mathrm{GeV}^{2}$. Dashed lines are obtained assuming Eq. (37), solid lines assuming Eq. (38). Note that the last $z_{1}$ bin in our calculation is narrower than in the corresponding experimental measurement.

For kaons, the same considerations lead to the following assumptions

$$
\begin{aligned}
& H_{1\left(u \rightarrow K^{-}\right)}^{\perp(1 / 2)}=-H_{1\left(u \rightarrow K^{+}\right)}^{\perp(1 / 2)}, \\
& H_{1\left(\bar{s} \rightarrow K^{-}\right)}^{\perp(1 / 2)}=-H_{1\left(\bar{s} \rightarrow K^{+}\right)}^{\perp(1 / 2)}, \\
& H_{1\left(d \rightarrow K^{-}\right)}^{\perp(1 / 2)}=-H_{1\left(d \rightarrow K^{+}\right)}^{\perp(1 / 2)}=0 .
\end{aligned}
$$

In Fig. 7 we show the values of the pion azimuthal asymmetry for four different ranges of $z_{1}$, as a function of $z_{2}$. The dashed curves and solid curves are obtained respectively under the assumptions in Eq. (37) and Eq. (38), respectively. The upper curves exceed the data for the higher $z_{2}$ values, which either reflects the need for corrections to the linear approximation in Eq. (40), or more likely that assuming no evolution for the Collins function may be too severe an approximation.

We calculated the corresponding $K K$ asymmetry, Fig. 8 and obtained even larger values, suggesting that there will be more dramatic effects in this accessible channel.

\section{CONCLUSIONS}

In this paper, we performed a new calculation of the Collins fragmentation function for $u \rightarrow \pi^{+}$, along the lines of Refs. [11, 12] but with some important differences: (i) we assumed the mass of the spectator is different from the mass of the fragmenting quark, (ii) we introduced a form factor at the hadron-quark vertex, (iii) we fitted the values of the model parameters to reproduce the unpolarized fragmentation function $D_{1}$ at a scale $Q_{0}^{2}=0.4 \mathrm{GeV}^{2}$. We compared the results of our model calculation to the available parametrizations of the Collins function [6, 7] extracted from $e^{+} e^{-}$annihilation and SIDIS data and found a reasonable agreement. We stressed the importance of critically considering different assumptions on the evolution of the Collins function with the energy scale.

For the first time we presented an estimate of the Collins function for $u \rightarrow K^{+}$and $\bar{s} \rightarrow K^{+}$. In particular, we found that the ratio $H_{1}^{\perp(1 / 2)} / D_{1}$ for $u \rightarrow K^{+}$is almost identical to that for $u \rightarrow \pi^{+}$, while the ratio for $\bar{s} \rightarrow K^{+}$is about twice as big.

Using the results of our model, we presented estimates for pion and kaon Collins asymmetries in $e^{+} e^{-}$annihilation at the BELLE experiment. In order to calculate the unfavored Collins functions we adopted the "strong interpretation" 


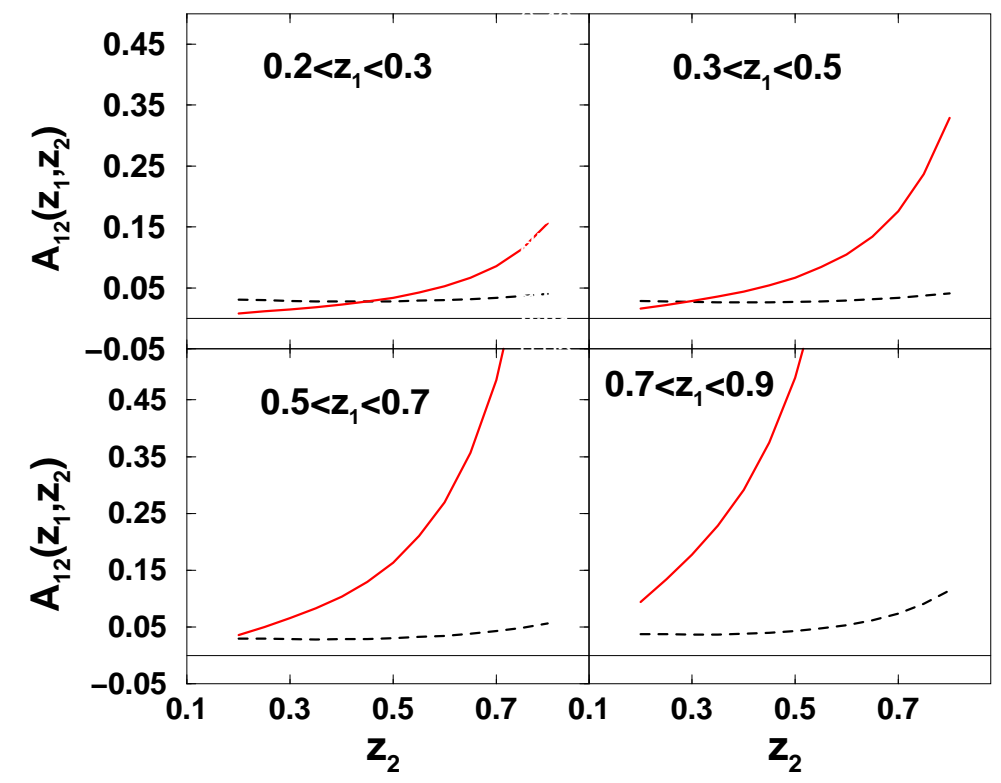

FIG. 8: Azimuthal asymmetry $A_{12}\left(z_{1}, z_{2}\right)$ for the production of two kaons as a function of $z_{2}$ and integrated in bins of $z_{1}$ at $Q^{2}=110.7 \mathrm{GeV}^{2}$. Dashed lines are obtained assuming Eq. (37), solid lines assuming Eq. (38).

of the Schäfer-Teryaev sum-rule [35]. Our results are in qualitative agreement with the available BELLE data on the pions, but large uncertainties arise from making different assumptions on the evolution of the Collins function as well as from determining the unfavored Collins fragmentation function. For the kaons, we predict the asymmetries to be larger than the pions.

\section{Acknowledgments}

The work of L. G and G.R.G. are supported in part by the U.S. Department of Energy under contracts DE-FG0207ER41460 and DE-FG02-92ER40702 respectively. A.M. wishes to thank the hospitality of the DESY Theory Group, where part of the present work was carried out.

[1] J. C. Collins, Nucl. Phys. B396, 161 (1993), hep-ph/9208213.

[2] A. Airapetian et al. (HERMES), Phys. Rev. Lett. 94, 012002 (2005), hep-ex/0408013.

[3] E. S. Ageev et al. (COMPASS), Nucl. Phys. B765, 31 (2007), hep-ex/0610068.

[4] K. Abe et al. (BELLE), Phys. Rev. Lett. 96, 232002 (2006), hep-ex/0507063.

[5] W. Vogelsang and F. Yuan, Phys. Rev. D72, 054028 (2005), hep-ph/0507266.

[6] A. V. Efremov, K. Goeke, and P. Schweitzer, Phys. Rev. D73, 094025 (2006), hep-ph/0603054.

[7] M. Anselmino et al., Phys. Rev. D75, 054032 (2007), hep-ph/0701006.

[8] A. Bacchetta, R. Kundu, A. Metz, and P. J. Mulders, Phys. Lett. B506, 155 (2001), hep-ph/0102278.

[9] A. Bacchetta, R. Kundu, A. Metz, and P. J. Mulders, Phys. Rev. D65, 094021 (2002), hep-ph/0201091.

[10] L. P. Gamberg, G. R. Goldstein, and K. A. Oganessyan, Phys. Rev. D68, 051501(R) (2003), hep-ph/0307139.

[11] A. Bacchetta, A. Metz, and J.-J. Yang, Phys. Lett. B574, 225 (2003), hep-ph/0307282.

[12] D. Amrath, A. Bacchetta, and A. Metz, Phys. Rev. D71, 114018 (2005), hep-ph/0504124.

[13] P. Schweitzer and A. Bacchetta, Nucl. Phys. A732, 106 (2004), hep-ph/0310318.

[14] L. P. Gamberg, D. S. Hwang, and K. A. Oganessyan, Phys. Lett. B584, 276 (2004), hep-ph/0311221.

[15] L. P. Gamberg (2004), hep-ph/0412367.

[16] M. Diefenthaler (HERMES), AIP Conf. Proc. 915, 509 (2007), hep-ex/0612010.

[17] F. Bradamante (COMPASS), AIP Conf. Proc. 915, 513 (2007), hep-ex/0702007.

[18] A. Bacchetta, U. D'Alesio, M. Diehl, and C. A. Miller, Phys. Rev. D70, 117504 (2004), hep-ph/0410050. 
[19] A. Bacchetta, M. Diehl, K. Goeke, A. Metz, P. J. Mulders, and M. Schlegel, JHEP 02, 093 (2007), hep-ph/0611265.

[20] A. Metz, Phys. Lett. B549, 139 (2002), hep-ph/0209054.

[21] J. C. Collins and A. Metz, Phys. Rev. Lett. 93, 252001 (2004), hep-ph/0408249.

[22] X. Ji and F. Yuan, Phys. Lett. B543, 66 (2002), hep-ph/0206057.

[23] A. V. Belitsky, X. Ji, and F. Yuan, Nucl. Phys. B656, 165 (2003), hep-ph/0208038.

[24] D. Boer, P. J. Mulders, and F. Pijlman, Nucl. Phys. B667, 201 (2003), hep-ph/0303034.

[25] X. Ji and Z. Zhu (1993), hep-ph/9402303.

[26] J. T. Londergan, A. Pang, and A. W. Thomas, Phys. Rev. D54, 3154 (1996), hep-ph/9604446.

[27] R. Jakob, P. J. Mulders, and J. Rodrigues, Nucl. Phys. A626, 937 (1997), hep-ph/9704335.

[28] H. Kitagawa and Y. Sakemi, Prog. Theor. Phys. 104, 421 (2000).

[29] S. Kretzer, Phys. Rev. D62, 054001 (2000), hep-ph/0003177.

[30] M. Hirai, S. Kumano, T. H. Nagai, and K. Sudoh, Phys. Rev. D75, 094009 (2007), hep-ph/0702250.

[31] A. Bacchetta, M. Boglione, A. Henneman, and P. J. Mulders, Phys. Rev. Lett. 85, 712 (2000), hep-ph/9912490.

[32] M. Gluck, E. Reya, and A. Vogt, Eur. Phys. J. C5, 461 (1998), hep-ph/9806404.

[33] A. A. Henneman, D. Boer, and P. J. Mulders, Nucl. Phys. B620, 331 (2002), hep-ph/0104271.

[34] D. Boer, R. Jakob, and P. J. Mulders, Phys. Lett. B424, 143 (1998), hep-ph/9711488.

[35] A. Schafer and O. V. Teryaev, Phys. Rev. D61, 077903 (2000), hep-ph/9908412. 